\title{
Geschlecht und Differenz in Organisationen: Von Gleichstellungspolitik und erfolgreichem Organisationslernen
}

Einige Forderungen der Frauenbewegung sind inzwischen in der „Mitte der Gesellschaft“ angekommen. Die Vereinbarkeitsproblematik oder die Unterrepräsentanz von Frauen in Führungspositionen werden als Problem anerkannt. Gender Mainstreaming oder Gender Diversity sollen in Unternehmen, Verwaltungen oder Schulen Entscheidungsprozesse und -routinen aufbrechen, um Ungleichbehandlungen zwischen den Geschlechtern abzubauen. Verändern Gender Mainstreaming oder Gender Diversity tatsächlich Prozesse und Strukturen in Organisationen zugunsten der Gleichbehandlung der Geschlechter? Wie setzen Organisationen solche Maßnahmen um? Und unter welchen Umständen sind sie überhaupt in der Lage, grundlegende Veränderungen im Geschlechterverhältnis herbeizuführen?

\section{Einleitung}

In die Geschlechterfrage ist Bewegung gekommen: Inzwischen werden geschlechtsspezifische Benachteiligungen auf dem Arbeitsmarkt, in Politik, Medien und Unternehmen eingestanden und der Handlungsbedarf wird anerkannt. Dies gilt zumindest für Forderungen wie die nach mehr Krippenplätzen, gleicher Bezahlung oder danach, Maßnahmen gegen die Unterrepräsentanz von weiblichen Führungskräften zu ergreifen. Diese Themen finden sich in Programmen von Parteien und Gewerkschaften und werden in der politischen Sphäre verhandelt. Weniger akzeptiert und heftiger umstritten sind dagegen Themen wie die missliche Situationen von Migrantinnen auf dem Arbeitsmarkt, von Hartz IV-Empfängerinnen oder von weiblichen Beschäftigten auf schlecht bezahlten Erwerbsarbeitsplätzen am unteren Ende der betrieblichen Hierarchien.

Innerhalb der Berufswelt sind die Optionen für Frauen mittlerweile vielfältiger geworden, allerdings lässt sich auch hier kein einheitliches Bild zeichnen. Gegenläufige und ambivalente Entwicklungen zwischen Wandel und Veränderungsresistenzen stehen nebeneinander. Auf der einen Seite gibt es Anzeichen dafür, dass es nicht mehr selbstverständlich und unhinterfragt hingenommen wird, hoch qualifizierte Beschäftigtengruppen aufgrund des Geschlechts aus betrieblichen Führungspositionen auszuschließen. Auf der anderen Seite zeigt ein Blick in die Arbeitsmarkt- und Berufsstatistiken, dass sich das bekannte Phänomen der geschlechtlichen Aufspaltung des Arbeitsmarktes weiterhin hartnäckig hält - oder sich gar unter veränderten ökonomischen Rahmenbedingungen wie auch im Zuge der Ausweitung prekärer Arbeitsbedingungen wieder verschärft. Trotz öffentlicher und politischer Debatten, formaler Gleichstellung, gleicher oder sogar besserer Bildungsabschlüsse, steigender Erwerbsquoten und gleicher Berufs- und Aufstiegsorientierung von Frauen arbeitet de facto die Mehrzahl der weiblichen Beschäftigten nach wie vor in Bereichen, die sich durch geringe Bezahlung, fehlende Aufstiegsmöglichkeiten und niedrige Aufstiegschancen auszeichnen. Prozesse eines vorsichtigen Wandels und deutliche Beharrungstendenzen lassen sich gleichzeitig beobachten.

Die bereits erwähnte „Bewegung in der Geschlechterfrage“ bedeutet somit auch, dass Ungleichheiten innerhalb der Gruppe der Frauen zunehmen: Einige, vorwiegend hoch qualifizierte Frauen können für sich neue gesellschaftliche Räume und Positionen erobern; andere verbleiben in marginalen Positionen oder werden ausgegrenzt. Auch diese Konstellation, die zunehmende Ausdifferenzierung von Ungleichheitslagen, fordert Gleichstellungspolitiken heraus.

\section{Die Rolle von Organisationen}

Organisationen, wie Unternehmen, Verwaltungen oder Schulen, sind zentrale
„Schaltstellen“ bei der Herstellung oder Relativierung von Geschlechterasymmetrien. Dass Geschlechtertrennungen in Organisationen deutlicher ausgeprägt sein können als auf dem Arbeitsmarkt insgesamt, belegen mittlerweile zahlreiche Studien (z. B. Acker 2006; Müller 2005; Wilz 2002; Allmendinger/Podsialkowski 2001). Beschäftigte bzw. Arbeitsuchende werden von Personalverantwortlichen für Arbeitsplätze ausgewählt und eingestellt, ihnen werden Weiterbildungsmaßnahmen angeboten, sie werden motiviert, befördert, versetzt oder demotiviert und entlassen. Annahmen über männliche und weibliche Lebensbereiche, über Kompetenzen, Eigenschaften und Verfügbarkeiten von Männern und Frauen fließen in die Praktiken, Routinen und Strukturen von Organisationen ein. Das aber bedeutet: Um grundlegende Wandlungsprozesse in Organisationen herbeizuführen, müssen genau diese Praktiken, Routinen und Strukturen auch grundlegend hinterfragt und verändert werden. Dabei gibt es große Unterschiede zwischen Organisationen - abhängig von der jeweiligen Organisationsstruktur und -kultur

Birgit Riegraf, PD Dr., Akademische
Oberrätin an der Fakultät für Soziologie der
Universität Bielefeld. Arbeitsschwerpunkte:
Theorien und Methodologien der Frauen-
und Geschlechterforschung; Arbeits- und
Organisationssoziologie; Staats- und Verwal-
tungsmodernisierung im internationalen
Vergleich; Wissensvermittlung zwischen
Theorie und Praxis.
email: birgit.riegraf@uni-bielefeld.de


(Größe, Differenzierung, Anerkennung von Gleichstellungsfragen), der Organisationsumwelt (Branche) oder den Organisationsregeln (Formalisierung, Gleichstellungsvorgaben). Ohne Kenntnisse darüber, wie einzelne Organisationen Gleichstellungsforderungen und -maßnahmen in ihre Praxis übersetzen, sind Prozesse des Wandels und der Beharrung am Arbeitsmarkt nicht zu verstehen.

Gleichstellungspolitiken und -strategien wie Gender Mainstreaming oder Gender Controlling zielen darauf ab, Entscheidungsprozesse und -routinen in Organisationen des öffentlichen Sektors, wie Verwaltungen oder Schulen, zu verändern. Im Gender Mainstreaming sollen alle an der politischen Gestaltung beteiligten Akteure bei allen Entscheidungsprozessen in allen Bereichen und auf allen Ebenen die Auswirkungen auf beide Geschlechter in den Blick nehmen. Während sich die Bundesregierung mit der Unterzeichnung des Amsterdamer Vertrages 1999 zur Übernahme von Gender Mainstraming verpflichtet hat, entspringt die Personal- und Organisationsentwicklungsstrategie des Managing Diversity der Praxis von Unternehmen. Managing Diversity wird inzwischen aber auch in anderen Organisationen, wie Verwaltungen oder Hochschulen, diskutiert und eingeführt. Im Managing Diversity sollen die Vielfalt und Heterogenität, die Beschäftigte aufgrund ihres Geschlechts, ihrer kulturellen und sozialen Herkunft oder sexuellen Orientierung in die Organisation einbringen, in Personal- und Organisationsentwicklungen anerkannt und gefördert werden. Diese Strategie wird inzwischen auch als möglicher Anknüpfungspunkt von Geschlechterpolitik diskutiert (DjbZ 2008; Rastetter 2007; Krell 2004; Koall 2001).

Allerdings: Im Gender Mainstreaming bleiben die zugrunde liegenden Vorstellungen von Geschlecht interpretationsoffen, und im Falle von Managing Diversity bleibt darüber hinaus unklar, auf welche Vorstellung von Differenz sich die Strategie bezieht. Dieser Beitrag will deutlich machen, was die konzeptionellen Unbestimmtheiten bedeuten: nämlich dass Organisationen in der Umsetzung von Gender Mainstreaming und Managing Diversity problemlos an unreflektierte geschlechterbezogene Stereotype und Polarisierungen anknüpfen können, die in Alltagsroutinen, Praktiken und Strukturen eingepasst sind. Somit bleiben die vielfältigen diskriminie- renden Zwänge und Behinderungen unangetastet, die sich in der alltäglichen Interaktion und Kommunikation, in symbolischen Ordnungen wie auch in Organisationsstrukturen insgesamt in vielfältiger Weise aufschichten. Deutlich werden soll auch, dass Strategien und Politiken wie Gender Mainstreaming und Managing Diversity mit Antidiskriminierungs- und Gerechtigkeitsdebatten verknüpft werden müssen (Riegraf 2006), weil sonst die Gefahr besteht, dass Geschlecht, Vielfalt und Differenz in einer Weise thematisiert werden, die die Ungleichheitslagen ebenfalls unangetastet lässt.

Im Folgenden wird der Blick nicht auf den vorsichtigen Wandel, sondern schwerpunktmäßig auf Beharrungstendenzen in Organisationen gerichtet. Im ersten Schritt wird gezeigt, wie sehr stereotype und polarisierende Differenzsetzungen zwischen Männern und Frauen sowie Hierarchisierungen den Praktiken, Routinen und Strukturen von Organisationen, den dortigen Interaktionen und Kommunikationen inhärent sind (Abschnitt 3). Im zweiten Schritt wird am Beispiel von Gender Mainstreaming und Managing Diversity dargestellt, inwieweit diese Politiken und Strategien in die Alltagsvorstellungen und -routinen von Geschlecht und Differenz und in die Organisationsstrukturen eingefügt werden können (Abschnitt 4). Schließlich wird diskutiert, unter welchen Bedingungen Organisationen überhaupt in der Lage sind, grundlegende Wandlungsprozesse im Geschlechterverhältnis einzuleiten, die Gleichstellungsforderungen gerecht werden (Abschnitt 5). Im Abschnitt 6 wird ein Fazit gezogen.

\section{Differenzsetzung und Hierarchisierungen in Organisation}

Organisationen sind in gesamtgesellschaftliche ökonomische Strukturen und Machtdynamiken eingebettet, die ihren Handlungsrahmen abstecken. Organisationsentwicklungen und -strategien werden jedoch nie vollständig durch solche Rahmungen bestimmt, sondern sie besitzen eigenständige Handlungsspielräume, die wiederum auf gesellschaftliche Bedingungen $\mathrm{zu}-$ rückwirken. Ungleichheitslagen nach Geschlecht, in der Überkreuzung mit Klasse oder Ethnie, sowie nach Alter oder Behinderung können von Organisationen - seien es Unternehmen, Verwaltungen oder Schulen -auf zwei Ebenen hergestellt, verstärkt, aber auch abgeschwächt werden: Erstens, auf der Ebene alltäglicher Interaktion und Kommunikation am Arbeitsplatz. Hier wirken Annahmen über Männer und Frauen, Männer- und Frauenbilder, teils verdeckt, aber gleichwohl virulent; zweitens, auf der Ebene von Organisationsstrukturen, z. B. über familienfreundliche oder familienfeindliche Arbeitszeitregelungen. Beide Ebenen besitzen zwar jeweils ihre eigene Bedeutung, Logik und Dynamik, sie stehen aber ebenfalls in einer engen und unübersehbaren Wechselwirkung (BeckerSchmidt 2007, S. 60ff.).

Auf der Ebene der alltäglichen Interaktion und Kommunikation zwischen Organisationsmitgliedern werden Ungleichheiten und Differenzierungen über polare Konstruktionen von sozialen Merkmalen, Eigenschaften oder Handlungsmustern hergestellt und markiert (positive Eigenschaften und Defizite; Fähigkeiten und Unfähigkeiten; Handlungspotenziale und Inkompetenzen), die sozialen Gruppen zugewiesen werden. Dies geschieht, wenn Managerinnen als besonders kommunikations-, team- und integrationsfähig klassifiziert werden und im Gegensatz dazu das Bild von den hierarchiegläubigen, durchsetzungsund konkurrenzbetonten Managern gezeichnet wird. Dies geschieht auch, wenn türkischen Mitarbeitern aufgrund von vermeintlichen Kompetenzen, Eigenschaften und Handlungspotenzialen andere Arbeitsbereiche als deutschen Mitarbeitern zugewiesen werden. In der alltäglichen Interaktion und Kommunikation, z. B. in Arbeitsund Projektgruppen, werden Kompetenzen, Eigenschaften und Handlungspotenziale nach Geschlecht, Ethnizität oder Klassen über symbolische und sprachliche Anordnungen oder Metaphern entwickelt, bestätigt und reproduziert. Die Stereotype und polarisierenden Zuschreibungen werden realitäts- und wirkungsmächtig, obwohl alle Beteiligten zugleich immer auch wissen oder zumindest ahnen, dass die $\mathrm{Zu}$ schreibungen keineswegs für alle Managerinnen und Manager oder für alle Türken oder Deutsche und auch nicht in allen Situationen zutreffen (Villa 2005). Der Bezug auf Stereotype und Polarisierungen in der alltäglichen Interaktion und Kommunikation hat im Rahmen von Organisationen durchaus System: In komplexen Situatio- 
nen mit geringer Erwartungssicherheit, z. B. wenn Entscheidungen schnell getroffen werden müssen und die gesamte Situation unübersichtlich ist, erlauben polarisierende Unterscheidungen zwischen Ähnlichkeiten und Differenzen den Akteuren eine schnelle Orientierung. Sie bieten einen greifbaren Ansatzpunk, um Komplexität zu reduzieren. Und damit sind sie auch ein Ausgangspunkt für Hierarchisierungen (Wilz 2002; Weinbach/Stichweh 2001). Diese Ebene steht in einer engen Verbindung zur Ebene der Organisationsstruktur.

Auf organisationsstruktureller Ebene entstehen Ungleichheiten und Differenzierungen z. B. über Stellenbeschreibungen, über Arbeitszeitregelungen oder Arbeitsplatzgestaltungen (Allmendinger/Podsiadlowski 2001; Heintz et al. 1997; Riegraf 1995). Dies geschieht beispielsweise, wenn in transnationalen Unternehmen neue Managementkonzepte zu örtlich und zeitlich ungebundenen und hoch arbeitsaufwendigen (zeitlich befristeten) Projektarbeiten führen (Boltanski/Chiapello 2003, S. 100186). Die nach wie vor ungleiche Verteilung von Versorgungsaufgaben pflegebedürftiger Angehöriger oder Kinder führt üblicherweise dazu, dass die entsprechenden Projektteams geschlechtsspezifisch zusammengestellt werden. Auf diesem Wege werden Managementtätigkeiten wieder zu einer „männlichen“ Domäne.

Wechselwirkungen und Verschränkungen zwischen der Ebene alltäglicher Interaktion und Kommunikation und der Organisationsstruktur werden deutlich, wenn die Konstruktion von geschlechterbezogenen Kompetenzen, Eigenschaften und Handlungspotenzialen als Legitimationsbasis für Ungleichbehandlungen aufgegriffen wird, die sich aus der Organisationsstruktur ergeben. Über Stereotypisierungen und Polarisierungen kann das Statusgefälle in Organisationen bestätigt, plausibilisiert und bekräftigt werden. Umgekehrt fließen die Distinktionen in organisationsstrukturelle Vorgaben ein und helfen, Privilegien zu sichern. Bereits Weber betont bei der Diskussion von Schließungsprozessen in Organisationen, dass „jedes noch so äußerliche Merkmal“ (gemeint hat er z. B. Sprache, soziale und kulturelle Herkunft, Religion) Ansatzpunkt für den Ausschluss von Konkurrenten sein kann (Weber 1980). Die Zuweisungen werden von denjenigen verteidigt, die Nutzen daraus ziehen (vgl. hierzu: Becker-Schmidt 2007, S. 60ff.).

\section{Geschlechter in gleichstellungspolitischen Maßnahmen}

Gemessen am programmatischen Anspruch sind Strategien wie Gender Mainstreaming eine Weiterentwicklung „traditioneller" Frauenförderpläne: Gender steht bekanntlich für die Abkehr von der Idee einer besonders „förderungswürdigen Problemgruppe Frau" hin zu Politiken und Strategien, die beide Geschlechter als Zielgruppe von Personal- und Organisationspolitiken ins Visier nehmen. Die Nischenpolitik soll durch eine Querschnittspolitik flankiert werden. Nicht mehr allein Gleichstellungsbeauftragte werden für Gleichstellungspolitiken verantwortlich gemacht. Und: Diversity Management stellt nicht mehr allein und explizit Geschlecht ins Zentrum, sondern Geschlecht soll als eine unter mehreren Kategorien berücksichtigt werden. Mit diesem gedanklichen Ansatz lässt die Organisations- und Personalstrategie Anschlussfähigkeit zur Diskussion über differente Arbeits- und Lebenslagen von Frauen vermuten. Wie aber sieht die praktische Umsetzung aus? Im Folgenden werden Umsetzungsschwierigkeiten von Gender Mainstreaming und Diversity Management in Organisationen vor dem Hintergrund der bisherigen Ausführungen genauer betrachtet.

\subsection{GENDER MAINSTREAMING}

Ein potenzielles Problem, das auch in der wissenschaftlichen Diskussion über Gender Mainstreaming diskutiert wird, ist der Verlust des emanzipatorischen Impetus von Gleichstellungspolitik. Hierzu kommt es, wenn in Organisationen - und damit „vor Ort" - an ein verkürztes Verständnis von Geschlecht angeknüpft wird (Bock et al. 2005). Die Wurzeln der Entstehung sozialer Ungleichheiten und Differenzierungen zwischen den Geschlechtern, ihre jeweiligen bereichsspezifischen Implikationen (beispielsweise für den Städtebau oder kommunale Haushalte) und das normative Ziel der Geschlechtergerechtigkeit werden dann nicht mehr oder höchstens noch am Rande thematisiert (Bereswill 2004; Meuser 2004). Eine verkürzte Umsetzung der Gender Mainstreaming-Strategie erfolgt, wenn geschlechtliche Zuweisungen nicht hinterfragt werden. Stattdessen wird bei Entscheidungs- und Gestaltungsprozessen schlicht auf leichter handhabbare, vorreflexive, erfahrungsbasierte Stereotype über Geschlechter Bezug genommen, die ohnehin die Interaktionen, Kommunikationen und Routinen von Organisationen prägen. Dies geschieht unter anderem, wenn mehr oder weniger explizit an Vorstellungen des weiblichen Arbeitsvermögens anknüpft wird, wonach Männer und Frauen unterschiedliche Kompetenzen, Eigenschaften und Handlungspotenziale haben.

Es kommt einem Teufelskreis gleich: Dualistische Geschlechterbilder und -konstruktionen, Festlegungen und Stereotypisierungen von Geschlechterdifferenz lassen sich unkompliziert und konfliktlos in das Alltagswissen der Akteure, in die Alltagsroutinen und die Strukturen von Organisationen einpassen. Sie werden auf diesem Wege zugleich erneut verfestigt, aktualisiert und reproduziert und bieten Anknüpfungspunkte für weitere Hierarchisierungen zwischen den Geschlechtern (Riegraf/Zimmermann 2005).

Illustrieren ließe sich dieses Problem an vielfältigen Beispielen. Pars pro toto sei hier nur auf zwei Fälle aus der Jugendarbeit und dem Städtebau verwiesen: In einem Papier, dass im Rahmen der Einführung von Gender Mainstreaming in der geschlechtsdifferenzierten Jugendarbeit 2001 in Berlin erstellt wurde, heißt es: „Leitbild: Wir erweitern unseren Blick auf die Geschlechterperspektive und fördern dadurch das Gleichgewicht von männlichen und weiblichen Persönlichkeiten in der Gesellschaft". Zu den Grundsatzzielen der Arbeit gehört demnach das „Erkennen von weiblichen und männlichen Persönlichkeitsanteilen (Anima/Animus)" (Jugendamt Treptow-Köpenick 2001, S.1; Brodtmann 2003). In diesem Verständnis von Gender Mainstreaming werden Differenz erhaltende und polarisierende Vorstellungen von Geschlecht unreflektiert übernommen und aktualisiert, die das Geschlechterverhältnis nicht grundlegend infrage stellen, sondern die sich problemlos in die Alltags- und Handlungsroutinen von Mitarbeitern und Mitarbeiterinnen in Organisationen einfügen lassen (Bock et al. 2005). Nichts anderes geschieht - Beispiel Städtebau -, wenn bei der Gestaltung von Grünanlagen oder Parks unreflektiert davon ausgegangen wird, dass alle Mädchen sich Rückzugsorte und geschützte Treffpunkte wünschen, wäh- 
rend das Interesse an Bewegung und Bolzplätzen einzig den Jungen, und zwar allen, zugeschrieben wird. Auch das Thema Sicherheit in öffentlichen Räumen wird ohne eine genaue Analyse einzig den Frauen zugeordnet, ohne z. B. zu reflektieren, dass männliche Homosexuelle in gleicher Weise massiv von aggressiven Handlungen betroffen sein können. Auch hier führt jeweils der fehlende Bezug auf theoretische Analysen über die Wurzeln geschlechterdifferenten Verhaltens zu traditionellen Festschreibungen von Geschlechterstereotypen.

\subsection{DIVERSITY MANAGEMENT}

Die Personalpolitik und Organisationsstrategie des Diversity Management findet sich in großen Unternehmen, aber zunehmend auch in Verwaltungen, Schulen, Non Governmental Organisationen (NGOs) und Universitäten. Managing Diversity soll zu Win-Win-Situationen führen. Es gilt die Annahme, dass der Abbau von Missachtung und erweiterte Chancen von Beschäftigten mit höheren Unternehmensgewinnen einhergehen. Das weibliche, multiethnische, multinationale, religiöse oder homosexuelle Humankapital wie auch unterschiedliche Erfahrungen - beispielsweise aufgrund des Alters - sollen anerkannt, erschlossen und integriert werden. Begründet wird dies mit den folgenden Argumenten:

- Die Anerkennung von Vielfalt und Heterogenität in Arbeitsgruppen führe zu steigender Kreativität und Problemlösungskompetenz;

- die Kosten würden sinken, da ein besseres Arbeitsklima und eine günstigere Umgebung zu geringerer Personalfluktuation führten;

- insgesamt würde das Unternehmen damit den Anforderungen an die Internationalisierung von Unternehmen, von Märkten und an die kulturelle Differenzierung von Kunden gerecht werden.

In den Selbstdarstellungen von Organisationen endet die Aufzählung von Unterschieden, Heterogenität und Vielfalt bisweilen in einem verräterischen „et cetera“. $\mathrm{Da}$ aber liegt bereits die Crux, denn: $\mathrm{Ob}$ sich Diversity Management tatsächlich als Anknüpfungspunkt für Gleichstellungspolitiken eignet, hängt im Wesentlichen davon ab, präzise benennen zu können, welche Heterogenität, welche Vielfalt und wel- che differenten Gruppen eigentlich genau gemeint sind. $\mathrm{Zu}$ überprüfen ist weiterhin: Werden alle Differenzen als Ungleichheit behandelt? Werden alle Dimensionen von Differenz und Ungleichheit in allen Situationen in Organisationen gleichermaßen und immer berücksichtigt? Und wie genau werden die Überschneidungen von Geschlecht mit anderen Ungleichheiten und Differenzierungen gedacht?

Managing Diversity ist - und das verweist auf das zweite Problem - sehr voraussetzungsvoll, weil es Differenzen und Ungleichheiten (z. B. bei Stellenbesetzungsverfahren und beim Stellenwechsel) markiert, die bislang in Organisationen als irrelevant zu gelten hatten: „Diversity verfolgt das Ziel, Menschen mit all ihren Unterschieden zu berücksichtigen, also nicht so zu tun, als seien sie (auf irgendeiner Ebene) gleich" (Stuber 2004, S. 20). Geschlecht, Ethnie, Religion oder Sexualität wurden bislang in den privaten Bereich verwiesen und werden nun - um Begrifflichkeiten aus der Systemtheorie zu bemühen - vom Bereich des Privaten in den Bereich des Dienstlichen verschoben. Aussagen wie „Bei uns spielt es keine Rolle, ob Sie Mann oder Frau, Deutsche oder Ausländerin, hetero- oder homosexuell sind“ stehen dem Diversity-Gedanken insofern entgegen, als es faktisch sowohl für den Einzelnen als auch für die Organisation natürlich (auf irgendeiner Ebene) einen Unterschied macht. Dies erkennt Diversity an (ebd, S. 20). Insofern gelangen mit Managing Diversity zu Recht wissenschaftliche Erkenntnisse über den Zusammenhang zwischen Geschlecht und Organisation ins Blickfeld, die darauf hinweisen, dass die Kategorie Geschlecht in Organisationen - entgegen der bisherigen Selbstdarstellung von Organisationen - relevant ist. Allerdings wird auf Differenzen und soziale Ungleichheiten vielfach in einer Art und Weise Bezug genommen, als entstünden sie außerhalb von Organisationen, als würden sie täglich von den Beschäftigten zur Arbeit in Form von Kompetenzen, Eigenschaften und Handlungspotenzialen quasi mitgebracht und sich dann in einem spezifischen Arbeitsstil niederschlagen, der sich aufgrund der Zugehörigkeit zu einem Geschlecht oder einer Nationalität herausgebildet hat (Frohnen 2005). Insofern wird die aktive Rolle, die Organisationen bei der Herstellung sozialer Ungleichheiten und Differenzierungen spielen, wiederum nicht mitbedacht.
Die dritte Schwierigkeit: Managing Diversity orientiert sich an den Zielen der jeweiligen Organisationen, was im Fall von Unternehmen wirtschaftliche Ziele sind. Dies bringt Elisabeth Girg, Mitarbeiterin im Global Diversity Team der Deutschen Bank in Frankfurt, in der Frankfurter Allgemeinen Zeitung vom September 2007 deutlich auf den Punkt: „Um keine Missverständnisse aufkommen zu lassen: Diversity ist kein Sozialthema, sondern ein geschäftsorientiertes Prinzip“. Sie begründet: „Wir wollen die besten Mitarbeiter am Markt ohne Rücksicht auf äußere Faktoren " (Obermeier 2007). Aber was geschieht, wenn sich keine Win-Win-Situation zwischen den Zielen des Unternehmens und den vielfältigen, gegebenenfalls auch heterogenen Interessen der Mitarbeiterinnen und Mitarbeiterin einstellt? Wenn ein Verkaufsgespräch aufgrund des Geschlechts der Beteiligten, ihrer kulturellen und sozialen Herkunft nicht reibungslos funktioniert? Welche Dimensionen von Heterogenität und Vielfalt werden in genau welchen Situationen im Unternehmen auf welche Weise anerkannt und gefördert? Anders formuliert: Welche Dimensionen von Heterogenität und Vielfalt sind in welchen Situationen in Organisationen eher nicht erwünscht? Welche Differenzen, soziale Ungleichheiten und Abwertungen werden im Rahmen von Organisationen erst hergestellt? Solche kritischen und Interessenwidersprüche reflektierenden Fragen werden in der Regel nicht gestellt. Die Aufmerksamkeit wird auf die Betonung von Heterogenität, Vielfältigkeit und Differenz konzentriert, was letztlich auch Ungleichheitslagen im Geschlechterverhältnis zu überlagern droht. Das zeigen folgende Auslassungen in der Diskussion und Konzeption:

(1) Die Anerkennung und Förderung von Differenzen ist keineswegs ein harmonisches Miteinander, sondern dies bedeutet, Privilegien elitärer und dominanter Gruppen abzubauen. Hinter Differenzen können sich handfeste Interessensgegensätze verbergen.

(2) Eine heterogene Gruppenzusammensetzung führt nicht unbedingt - und schon gar nicht per se - zu einem produktiven Miteinander. Es können unproduktive Blockaden aufgrund differenter Interessen entstehen. Nicht alle Differenzen und Ungleichheiten lassen sich einfach in betriebswirtschaftliche Gewinne ummünzen. 
(3) Weiterhin besteht die Gefahr von essentialistischen und stereotypen Zuschreibungen, so z. B. wenn weibliche Führungskräfte als Trägerinnen „typischer weiblicher Verhaltensweisen" eingesetzt werden (vgl. Krell 2004).

(4) Und schließlich können Organisationen die Vielfalt von Organisationsmitgliedern nicht allein zum Ausgangspunkt von Analysen und Maßnahmen machen. Nichtanerkennungsmomente, aufgrund von Alter, Ethnie, Geschlecht, werden über Praktiken in Organisationen erst hergestellt.

Aufgrund der Vagheit des Diversity Konzepts können je nach Situationen und Konstellationen von Mitarbeitern Stereotype und Polarisierungen in der alltäglichen Interaktion und Kommunikation wahlweise aktualisiert oder ausgeblendet werden. Auf diesem Wege passt sich Diversity reibungslos in die Praktiken, Routinen und Strukturen von Organisationen ein, ohne diese zu hinterfragen oder gar zu verändern.

\section{Organisationslernen und Gleichstellungspolitik}

Die Probleme, die sich bei der Anwendung und Umsetzung von Gender Mainstreaming und Diversity Management zeigen, sind - und das sollte nochmals betont werden - weder zufällig noch willkürlich. Sie sind struktureller Natur, da in Organisationen Vorstellungen von Geschlecht oder Ethnizität in die Handlungsroutinen und Organisationsstrukturen eingewoben sind. Gleichstellungsstrategien, deren Ziel es ist, die gesellschaftlichen Ungleichheits- und Diskriminierungsmechanismen abzubauen, erfordern einen Bruch mit bisherigen Routinen, Praktiken und Strukturen und komplexe Reflexionsprozesse. Voraussetzung ist eine umfassende Organisationsanalyse. Sie muss offen legen, welche Praktiken und Regeln, welche symbolischen Ordnungen oder auch Metaphern und Strukturen die Stellungen nach Geschlecht, Ethnizität oder Klassen überhaupt erst hervorbringen. Und sie muss zeigen, welche Relationen sie annehmen, welchen Stellenwert sie innerhalb des organisationalen Gefüges haben, zu welchen Wechselwirkungen sie führen.
Studien zum Organisationslernen geben Aufschluss darüber, unter welchen Bedingungen Organisationen in der Lage sind, sich einer grundlegenden Selbstreflexion, z. B. im Umgang mit der Kategorie Geschlecht, zu unterziehen und Innovationen im Geschlechterverhältnis anzustoßen. Sie zeigen zugleich, weshalb sich wesentliche Wandlungsprozesse in Verwaltungen, Unternehmen oder Schulen nur sehr langsam, mit großer Zeitverzögerung und manchmal auch gar nicht durchsetzen und geben so Hinweise darauf, wie erfolgreiche Gleichstellungsstrategien aussehen können (Messner 2003; Riegraf 2000; Willke 1998; Mintzberg 1996; Wiesenthal 1995): Komplexes Lernen, grundsätzlicher Wandel, Strategien jenseits routinisierten Handelns sind für Organisationen risikobeladen, schwierig und voraussetzungsvoll. Gegenstand komplexen Lernens und radikaler Innovation von Organisationen ist vor allem das Wissenssystem, in dem operative Regeln, normative Prämissen und kognitive Annahmen gespeichert sind. Das Wissenssystem jedoch baut nicht nur auf Kenntnissen über objektive Ursache- und Wirkungsketten auf (die ja häufig unbekannt und unsicher sind), sondern auch auf Organisationsmythen, mit denen die Lücken empirischen und theoretischen Wissens gefüllt werden. Es beinhaltet auch „mental maps", die implizites Wissen komprimieren und „Landkarten in den Köpfen" darstellen, um überhaupt erst Orientierung in einer komplexen Welt und damit zielgerichtetes kollektives Handeln von Organisationen zulassen. Die Grenzen zwischen kognitiven Annahmen, normativen Ideen und Organisationsmythen sind folglich fließend. Komplexes Lernen und radikale Innovationen im Sinne weitreichender Strategiewechsel stellen gerade diese Wissens- und Deutungsmuster infrage, die für die Handlungsfähigkeit von Organisationen konstitutiv sind („core beliefs“) (Messner 2003).

Organisationen neigen dazu, auf Veränderungsanforderungen „von außen“ zunächst mit Ignoranz zu reagieren. Oder sie versuchen, eingeschliffene Handlungsmuster und Einstellungen durch Rechtfertigung zu verteidigen. Eine dritte Variante ist, dass sie eine selektive Wahrnehmung entwickeln, d.h. sie greifen ausschließlich diejenigen neuen Anforderungen auf, die an das etablierte Wissens-, Deutungs- und Regelsystem anschlussfähig sind. Anders formuliert: Handeln und Lernprozesse in
Organisationen sind aufgrund der Bedingungen kollektiven Handelns hochgradig pfadabhängig. Neues Wissen kann sich nur sukzessiv gegen vorhandene Wissens- und Deutungsmuster durchsetzen und als relevant identifiziert und akzeptiert werden. Als Ursache tief greifenden Wandels sind Zweifel an oder Erschütterungen von „bewährten Regeln“ und Orientierungen oder Inkonsistenzen zwischen neuem Wissen und Organisationsmythen nicht ausreichend. Weil Organisationen auf "geglaubte", internalisierte und routinisierte „mental maps“ angewiesen sind, müssen tief verankerte und lange Zeit eingeübte und erfolgreiche Orientierungssysteme erst nachhaltig entwertet und durch neue, überzeugende Orientierungsangebote ersetzt werden.

Zuschreibungen wie die nach Geschlecht sind in das organisationale Wissenssystem eingelassen. Punktuelle Gender Trainings im Falle des Gender Mainstreaming oder sogenannte Diversity Trainings im Falle des Diversity Managements wie auch eine Änderung des Unternehmensleitbildes sind gegenwärtig wohl die häufigsten gleichstellungspolitischen Maßnahmen in Organisationen. In den Trainings geht es vor allem um die Sensibilisierung für "Geschlecht" oder „Ethnie“, also für Unterschiede; es geht um Einstellungsänderungen im Sinne der Wertschätzung des jeweils anderen Geschlechts, es geht um Fertigkeiten wie beispielsweise darum, die Kommunikation mit Personen anderer kultureller Zugehörigkeit zu erlernen. Solche punktuellen Maßnahmen werden einfach in die Routinen, Praktiken und Strukturen eingepasst und sind bei Weitem nicht ausreichend, eine grundlegende Erschütterung lange eingeübter Orientierungssysteme zu bewirken. Und sie sind schon gar nicht geeignet, einen umfassenden Wandel in Organisationen einzuleiten. Die Diskussion zum Organisationslernen zeigt, dass mindestens zwei Voraussetzungen erfüllt sein müssen, um dies zu erreichen: Zum einen müssen Strategien auf eine längerfristige Perspektive ausgerichtet werden, damit sie erfolgreich sein können. Zum anderen müssen permanente und längerfristige Zweifel über bereits erreichte Fortschritte institutionalisiert werden. Das heißt: Auch Erfolge müssen daraufhin überprüft werden, ob sie wirklich frei von Widersprüchlichkeiten, Ambivalenzen und Mehrdeutigkeiten sind. Mit anderen Worten: Erfolgreiche 
Lernprozesse im Bezug auf Geschlecht sind nur über die Institutionalisierung längerfristiger, immer wieder selbstreflexiver Prozesse möglich - z. B. in Form prozessevaluierender Expertisen oder längerfristiger Beratungstätigkeiten, die nicht immer organisationsintern bewältigt werden können. Einzelne, isolierte Maßnahmen führen nicht zu längerfristigen Innovationen (Riegraf/Zimmermann 2005; Riegraf 2000).

Grundlegende Umstrukturierungsprozesse in Organisationen - auch das ist aus den Untersuchungen zum Organisationslernen bekannt - werden durch externe "Schocks" erleichtert, wenn also Organisationen in eine grundlegende Krise geraten und es ganz offensichtlich erkennbar wird, dass alte Orientierungsmuster nicht mehr greifen. In solchen Situationen kann es zu einem Wechsel in den Führungspositionen, zur Anerkennung von „Organisationsdissidenten“ oder zu hoher Experimentierbereitschaft kommen (Wiesenthal 1995; Riegraf 2000).

\section{0 \\ Fazit}

Organisationen verfügen über Handlungsspielräume. Sie können soziale Ungleichheiten und Differenzierungen nach Geschlecht, Klasse oder Ethnie, aber auch nach Alter oder Behinderung herstellen oder reproduzieren. Sie haben umgekehrt die Möglichkeit, solche Ungleichheiten und Differenzierungen durch gezieltes Organisationshandeln abzuschwächen.

Dualistische Vorstellungen von Mann und Frau, also Männer- und Frauenbilder, sind in Organisationsstrukturen und Orientierungssystemen wie ein Bodensatz eingelassen, sie werden in der alltäglichen Kommunikation und Interaktion zwischen Organisationsmitgliedern aktiviert und hergestellt. Maßnahmen wie Gender Mainstreaming oder Managing Diversity sollen Abhilfe schaffen. Jedoch: Ohne einen kritischen, reflektierten Bezug auf Geschlecht und Differenz fügen sie sich allzu geschmeidig und bruchlos in die Alltagsroutinen und Organisationsstrukturen ein. Die Diskussion zum Organisationslernen zeigt: Ohne längerfristig angelegte und kontinuierliche Organisationsentwicklungsstrategien bleiben auch Gender Mainstreaming und Diversity Management unwirksam. Doch wenn Ungleichheitslagen unangetastet bleiben, entfalten sie ungebrochen ihre Wirkung. Sie werden genutzt, um Statusungleichheiten in Organisationen zu legitimieren und abzusichern; organisationsinterne Handlungsspielräume zum Abbau von Asymmetrien bleiben unangetastet. Zugespitzt formuliert: Auf Gleichstellung bezogene Ansätze werden so zu einer Chimäre. Ohne einen ernsthaften Bezug zu Antidiskriminierungs- und Gerechtigkeitsdebatten und ohne die Bedingungen des Organisationslernens zu berücksichtigen, kommen gleichstellungspolitische Forderungen und Vorstellungen in Organisationen nicht aus.

\section{LITERATUR}

Acker, J. (2006): Inequality Regimes. Gender, Class, and Race in Organizations, in: Gender \& Society 4, S. 441-464

Allmendinger, J./Podsiadlowski, A. (2001): Segregation in Organisationen und Arbeitsgruppen, in: Heintz, B. (Hrsg.): Geschlechtersoziologie, Kölner Zeitschrift für Soziologie und Sozialpsychologie, Sonderheft 41, Opladen, S. 276-307

Becker-Schmidt, R. (2007): "Class", "gender", , ethnicity ", "race“: Logiken der Differenzsetzung, Verschränkung von Ungleichheitslagen und gesellschaftliche Strukturierung, in: Klinger, C./Knapp, G./Sauer, B. (Hrsg.): Achsen der Ungleichheit. Zum Verhältnis von Klasse, Geschlecht und Ethnizität, Frankfurt a. Main/New York, S. 56-83

Bereswill, M. (2004): "Gender" als neue Humanressource? Gender Mainstreaming und Geschlechterdemokratie zwischen Ökonomisierung und Gesellschaftskritik, in: Meuser, M./Neusüß, C. (Hrsg): Gender Mainstreaming. Konzepte, Handlungsfelder, Instrumente (Schriftenreihe der Bundeszentrale für politische Bildung, Band 418), Bonn, S. 52-70 Bock, S./Matthies, H./Riegraf, B./Zimmermann, K. (2005): Wissenskommunikation im Kontext von Gender Mainstreaming. Das Beispiel deutscher Kommunen, in: Ernst, S./Warwas, J./Kirsch-Auwärter, E. (Hrsg.): Wissenstransform. Wissenskommunikation in Netzwerken, Hamburg, S. $172-190$
Boltanski, I./Chiapello, E. (2003): Der neue Geist des Kapitalismus, Konstanz

Brodtmann, T. (2003): Gender Mainstreaming in der Jugendarbeit des Berliner Bezirks Treptow-Köpenick, Diplomarbeit, Technische Universität Berlin Fachbereich Erziehungswissenschaften, http://www.maktrep-koep.de/Dokumente/DiplomarbeitGM.pdf Zeitschrift des Deutschen Juristinnenverbandes (DjbZ) (2008): „Gender Mainstreaming und Managing Diversity: Alternativen zu Antidiskriminierungsrecht und Gleichstellungsgesetzen im Arbeitsleben?" Schwerpunktheft 1

Frohnen, A. (2005): Diversity-in-Action. Multinationalität in globalen Unternehmen am Beispiel Ford, Bielefeld

Heintz, B./Nadai, E./Fischer, R./ Ummel, H. (1997): Ungleich unter Gleichen, Frankfurt a. M./New York

Jugendamt Treptow-Köpenick (2001): Ziele der Arbeitsgruppe "geschlechtsdifferenzierte Jugendarbeit" , Leitbild, März 2001, Berlin Koall, I. (2001): Managing Gender und Diversity. Von der Homogenität zur Heterogenität in der Organisation der Unternehmung, Münster Krell, G. (2004): Managing Diversity: Chancengleichheit als Wettbewerbsfaktor, in: Krell, G. (Hrsg.): Chancengleichheit durch Personalmanagement, Wiesbaden, S. 41-56 
Messner, D. (2003): Wissenschaftliche Politikberatung. Einige Anmerkungen zu einem schwierigen Verhältnis, in: Hirscher, G./Korte, K.-R. (Hrsg.): Information und Entscheidung. Kommunikationsmanagement der politischen Führung, Wiesbaden

Meuser, M. (2004): Von der Frauengleichstellungspolitik zu Gender Mainstreaming: Organisationsveränderungen durch Geschlechterpolitik?, in: Pasero, U./Priddat, B. (Hrsg.): Organisationen und Netzwerke: Der Fall Gender, Wiesbaden, S. 93-112

Mintzberg, H. (1996): Managing Government - Governing Management, in: Harvard Buisness Review, Mai/Juni, S. 75-83

Müller, U. (2005): Geschlecht, Arbeit und Organisationswandel. Eine ReThematisierung, in: Kurz-Scherf, I./Corell, L./Janczyk, S. (Hrsg.): Arbeit: Zukunft, Münster, S. 224-240

Obermeier, B. (2007): Es lebe der Unterschied, Frankfurter Allgemeine Zeitung, FAZ-Net vom 17. November

Rastetter, D. (2007): Managing Diversity: Eine für die Gleichstellung von Frauen gewinnbringende Strategie?, in: aktuelle informationen 2,

S. $23-24$

Riegraf, B. (1995): Mikropolitik und Geschlecht, Opladen

Riegraf, B. (2000): Organisationswandel, Organisationslernen und das Geschlechterverhältnis, in: Lenz, I./Nickel, H. M./Riegraf, B. (Hrsg.): Geschlecht, Arbeit, Zukunft, Münster

Riegraf, B. (2006): Gerechtigkeitskonzeptionen im Wandel: Von der Gleichheit zur Differenz, in: Aulenbacher, B./Bereswill, M./Löw, M./ Meuser, M./Mordt, G./Schäfer, R./Scholz, S. (Hrsg.): Arbeiten mit der Kategorie Geschlecht. FrauenMännerGeschlechterforschung, Forum
Frauen- und Geschlechterforschung, Schriftenreihe der Sektion Frauenund Geschlechterforschung in der Deutschen Gesellschaft für Soziologie, Wiesbaden, S. 323-333

Riegraf, B./Zimmermann, K. (2005): Der Wandel der Wissensordnung in der Wissensgesellschaft und die Kategorie Geschlecht. Annäherungen an ein Forschungsdesiderat, in: Funder, M./Dörhöfer, S./Rauch, C. (Hrsg.): Jenseits der Geschlechterdifferenz? Geschlechterverhältnisse in der Informations- und Wissensgesellschaft, München und Mering, S. 21-37

Stuber, M. (2004): Das Potenzial von Vielfalt nutzen - Den Erfolg durch Offenheit steigern, Neuwied/Kriftel

Villa, P. (2005): Wer weiß was? Geschlechtersoziologische Überlegungen zum produktiven Scheitern konkreter Menschen bei der Subjektwerdung, in: Funder, M./Dörhöfer, S./Rauch, C. (Hrsg.): Jenseits der Geschlechterdifferenz? Geschlechterverhältnisse in der Informations- und Wissensgesellschaft, München und Mering, S. 39-57

Weber, M. (1980): Wirtschaft und Gesellschaft, Tübingen

Weinbach, C./Stichweh, R. (2001): Die Geschlechterdifferenz in der funktional differenzierten Gesellschaft, in: Heinz, B. (Hrsg.): Geschlechtersoziologie, Wiesbaden, S. 30-52

Wiesenthal, H. (1995): Konventionelles und unkonventionelles Organisationslernen: Literaturreport und Ergänzungsvorschläge, in: Zeitschrift für Soziologie 2, S. 137-155

Willke, H. (1998): Systemisches Wissensmanagment, Stuttgart

Wilz, S. (2002): Organisation und Geschlecht. Strukturelle Bindungen und kontingente Kopplungen, Opladen 